\title{
An Ultrasound Mediated Green Synthesis of Benzimidazolylthiounsaturatednitriles Using Water as a Green Solvent
}

\author{
Sadhu Srinivas Rao, Chittireddy Venkata Ramana Reddy, and Pramod Kumar Dubey \\ Department of Chemistry, College of Engineering, Jawaharlal Nehru Technological University Hyderabad, Kukatpally, \\ Hyderabad 500 085, India \\ Correspondence should be addressed to Sadhu Srinivas Rao; seenu604@gmail.com
}

Received 8 July 2014; Revised 27 September 2014; Accepted 20 October 2014; Published 9 November 2014

Academic Editor: Jonathan White

Copyright ( 2014 Sadhu Srinivas Rao et al. This is an open access article distributed under the Creative Commons Attribution License, which permits unrestricted use, distribution, and reproduction in any medium, provided the original work is properly cited.

Reaction of 2-cyanothiomethylbenzimidazole 1 with an aromatic aldehydes in water under ultrasonic irradiation for 10-13 min gave the corresponding unsaturated nitriles $\mathbf{2} \mathbf{a}-\mathbf{h}$ which is an efficient and simple method under green conditions. The unsaturated nitrile derivatives were obtained in $86-98 \%$ yield with a short reaction time without any tedious workup procedures.

\section{Introduction}

The importance of heterocycles in many fields of science (including organic, inorganic, bioorganic, agricultural, industrial, pharmaceutical, and medicinal chemistry, as well as material science) can hardly be overstated and justifies a long-lasting effort to work out new synthetic protocols for their production [1]. Ultrasound enhances the reactivity of molecules towards many chemical reactions via the process of acoustic cavitation [2]. The assistance of US irradiation efficiently shortens the reaction times. Simple experimental procedure, very good yields, increased selectivity, and clean reaction of many US-induced organic transformations offer additional convenience in the field of synthetic organic chemistry [3-8]. Recently, US has been utilized to accelerate a wide number of synthetically useful organic reactions $[9,10]$. In addition to the field of organic chemistry, sonochemistry has also been used in the preparation of micro- and nanomaterials (i.e., protein microspheres) [11].

The $\alpha, \beta$-unsaturated nitriles play an important role in drug discovery programs. Various unsaturated nitrile derivatives are also reported to exhibit a wide spectrum of biological properties such as cardiotonic agents. Analogue possessing $\alpha, \beta$-unsaturated nitriles at the $17-\beta$ position had high activity in natural products. In Digoxin, the unsaturated 17-lactone plays an important role in receptor binding. Saturation of the lactone ring dramatically reduced the biological activity. In Digoxin, if the lactone group at 17th position is replaced with $\alpha, \beta$-unsaturated nitriles ( $-\mathrm{C}=\mathrm{C}-\mathrm{CN}$ group), there is no loss in biological activity $[12,13]$ (see Figure 1).

Walker et al. reported [14] that condensation of 3-formyl$1 \mathrm{H}$-indole-2-carboxylate with 3-nitrophenyl acetonitrile in piperidine using ethanol under reflux conditions for $70 \mathrm{~h}$ gave the corresponding $\alpha, \beta$-unsaturated nitriles. Mukarram et al. reported [15] that the condensation of p-substituted benzaldehyde with active methylene compounds gave the corresponding unsaturated nitrile derivative, that is, entacapone, which is used for treatment of Parkinson's disease. Condensation of benzaldehyde with cyanoethyl acetate in ethanol at $\mathrm{RT}$ in the presence of a catalytic amount of Baker's yeast to give unsaturated nitriles was reported by Pratap et al. [16]. Al-Kadasi and Nazeruddin reported that the condensation of 4-hydroxycoumarin with aromatic aldehydes in water under ultrasound irradiations at ambient temperature gave excellent yield of the desired products in shorter reaction time [17]. Synthesis of 2-amino-4,6-diphenylnicotinonitriles via fourcomponent reaction of malononitrile, aromatic aldehydes, acetophenone derivatives, and ammonium acetate in water under ultrasound irradiation is described by Safari et al. [18].

Based on the few literature citations given above herein we report an ultrasound mediated synthesis of 

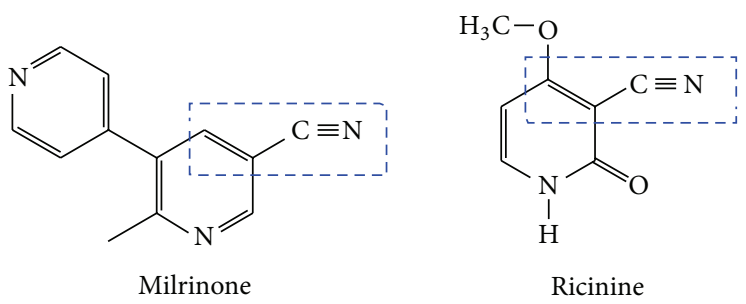<smiles>CCOC(=O)C1=C(O)C(O)C(C#N)=CC1=CC(=O)N(CC)CC</smiles><smiles>CC(C)(C#N)c1cc(Cn2cncn2)cc(C(C)(C)C#N)c1</smiles>

Figure 1<smiles>N#CCSc1nc2ccccc2[nH]1</smiles>

(1)<smiles>N#C/C(=C\[Al])Sc1nc2ccccc2[nH]1</smiles>

$(\mathbf{2 a}-\mathbf{h})$

Scheme 1: Synthesis of $\mathbf{2} \mathbf{a}-\mathbf{h}$ from $\mathbf{1}$ under sonochemical reaction.

$\alpha, \beta$-unsaturated nitriles, that is, 2-( $(1 \mathrm{H}$-benzimidazol-2yl)thio)-3-arylacrylonitrile in aqueous medium.

\section{Results and Discussion}

The use of ultrasonic waves is a convenient technique in organic synthesis; its development in the past few years has been considerably increased in an attempt to gain an insight into the mechanism of action inside the reaction flask. Several applications in organic synthesis have made sonochemistry attractive to many researchers and it is increasingly used in organic synthesis. It has proved to be a great tool for improving yields and decreasing the reaction time.

In our continued interest in the development of green methodologies and earlier studies [19], we report here the green synthesis of 2-cyanothiomethylbenzimidazoles from the Knoevenagel condensation of benzimidazole containing active methylene compounds with various aromatic aldehydes in the presence of water as a mild base.

Condensation of 2-thiobenzimidazoleacetonitrile with the appropriate aromatic aldehydes in water is carried out sonochemically; the reaction time was just $10-13$ min to yield the unsaturated nitriles of the corresponding benzimidazole thiols with high yields. In this reaction, water acts as the base to facilitate conversion of the active ethylene to a carbanion intermediate. The carbanion then attacks at the carbonyl compound of an aromatic aldehyde leading to the formation of a $\mathrm{C}-\mathrm{C}$ double bond following elimination of water as a byproduct (Scheme 1) (Table 1).

\section{Experimental}

3.1. General Considerations. All chemicals were purchased from commercial suppliers and used further without purification. IR Spectra were recorded with Jasca FT-IR 5300. ${ }^{1} \mathrm{H}$ $\mathrm{NMR}$ and ${ }^{13} \mathrm{C}$ NMR were recorded in $\mathrm{CDCl}_{3} / \mathrm{DMSO}$ using Varian 400-MHz instrument. Mass spectra were recorded on an Agilent LC-MS instrument giving only $\mathrm{M}^{+}$values in $\mathrm{Q}+$ 1 mode. Thin-layer chromatography (TLC) analyses were carried out on glass plates coated with silica gel GF-254 and visualization was achieved using iodine vapours or UV lamp. Ultrasound for sonication is generated with the help of ultrasonic instrument. The specifications, operating parameters, and the details of the set-up are as follows. Make: China; operating frequency: $36 \pm 3 \mathrm{kHz}$; rated output power: $700 \mathrm{~W}$; tank size: $240 \mathrm{~mm} \times 135 \mathrm{~mm} \times 100 \mathrm{~mm}$.

3.2. Synthesis of $\mathbf{2} \boldsymbol{a}-\boldsymbol{h}$ from $\mathbf{1}$ Using Sonochemical Method. To a mixture of $\mathbf{1}(10 \mathrm{mM})$, aromatic aldehyde $(10 \mathrm{mM})$ in water $(20 \mathrm{~mL})$ was added under sonication, by keeping all sonication parameters constant till the completion of the reaction. The reaction progress was monitored by TLC; after 
TABLE 1: Synthesis of $\mathbf{2 a}-\mathbf{h}$ from $\mathbf{1}$ using sonication.

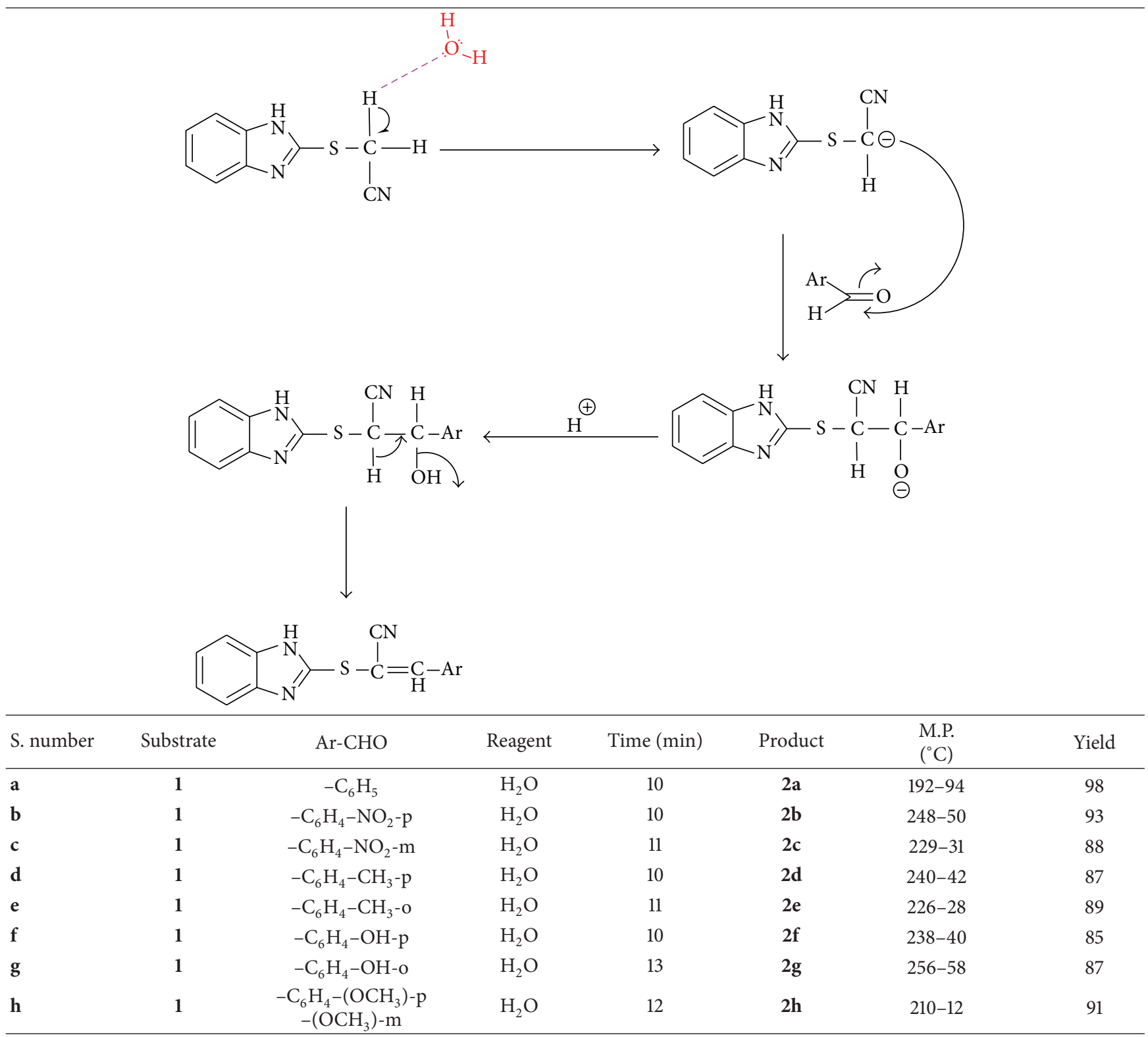

10-13 min, the reaction was found to be completed. The mixture was filtered and dried. The obtained crude product was recrystallized using ethyl acetate as solvent to obtain $\mathbf{2 a}-\mathbf{h}$. The reaction time was confirmed by repeating the procedure for three more times.

\subsection{Spectrometric Characterization of the Synthesized Compounds}

3.3.1. 2-((1H-Benzimidazol-2-yl)thio)-3-phenylacrylonitrile (2a). IR (KBr): $3400-2800 \mathrm{~cm}^{-1}$ (br, m, -NH-); ${ }^{1} \mathrm{H}-\mathrm{NMR}$ $\left(400 \mathrm{MHz}, \mathrm{DMSO}-\mathrm{d}_{6} / \mathrm{TMS}\right): \delta 8.20(\mathrm{~s}, 1 \mathrm{H}), 8.10(\mathrm{~d}, J=$ $4.6 \mathrm{~Hz}, 2 \mathrm{H}), 7.66-7.33(\mathrm{~m}, J=6.6 \mathrm{~Hz}, 3 \mathrm{H}), 7.65-7.34(\mathrm{~m}$, $J=6.4 \mathrm{~Hz}, 4 \mathrm{H}), 7.38-7.27(\mathrm{~m}, 2 \mathrm{H}), 10.6(\mathrm{~s}, 1 \mathrm{H},-\mathrm{NH}-),{ }^{13} \mathrm{C}$ NMR: $\delta 140.5,138.5,135.0,132.5,128.3,122.9,115.5,111.5$ ppm;
MS (CI): $m / z 278\left[\mathrm{M}^{\bullet+}+1\right]$. Anal. Calcd. for $\mathrm{C}_{16} \mathrm{H}_{11} \mathrm{~N}_{3} \mathrm{~S}: \mathrm{C}$, 69.29; H, 4.00; N, 15.15; S, 11.56. Found: C, 69.26; H, 3.96; N, 15.17; S, 11.59 .

3.3.2. 2-((1H-Benzimidazol-2-yl)thio)-3-(4-nitrophenyl)acrylonitrile (2b). IR (KBr): $3400-2800 \mathrm{~cm}^{-1}$ (br, m, $\left.-\mathrm{NH}-\right) ;{ }^{1} \mathrm{H}-$ NMR (400 MHz, DMSO- $\left.\mathrm{d}_{6} / \mathrm{TMS}\right): \delta 8.16(\mathrm{~s}, 1 \mathrm{H}), 8.02(\mathrm{~d}, J=$ $4.4 \mathrm{~Hz}, 2 \mathrm{H}), 7.62-7.31(\mathrm{~m}, J=6.5 \mathrm{~Hz}, 2 \mathrm{H}), 7.61-7.44(\mathrm{~m}, J=$ $6.5 \mathrm{~Hz}, 4 \mathrm{H}), 10.5$ (s, $1 \mathrm{H},-\mathrm{NH}-),{ }^{13} \mathrm{C}$ NMR: $\delta 141.3,137.5,134.0$, $131.5,127.3,121.9,116.5,110.5$ ppm; MS (CI): $m / z 323\left[\mathrm{M}^{\circ+}+1\right]$. Anal. Calcd. for $\mathrm{C}_{16} \mathrm{H}_{10} \mathrm{~N}_{4}$ OS: C, 59.62; H, 3.13; N, 17.38; O, 9.93; S, 9.95. Found: C, 59.64; H, 3.16; N, 17.36; O, 9.95; S, 9.97.

3.3.3. 2-((1H-Benzimidazol-2-yl)thio)-3-(3-nitrophenyl)acrylonitrile (2c). IR (KBr): $3400-2800 \mathrm{~cm}^{-1}$ (br, m, $-\mathrm{NH}-$ ); ${ }^{1} \mathrm{H}-\mathrm{NMR}$ (400 MHz, DMSO-d 6 /TMS): $\delta 8.21$ (s, $1 \mathrm{H}$ ), 8.16 
$(\mathrm{d}, J=4.4 \mathrm{~Hz}, 2 \mathrm{H}), 7.98-7.35(\mathrm{~m}, J=6.4 \mathrm{~Hz}, 2 \mathrm{H}), 7.60-7.43$ $(\mathrm{m}, J=6.5 \mathrm{~Hz}, 4 \mathrm{H}), 10.6$ (s, 1H, -NH-), ${ }^{13} \mathrm{C}$ NMR: $\delta 141.2$, $138.5,134.2,131.7,127.5,120.9,117.5,111.5$ ppm; MS (CI): $m / z$ $323\left[\mathrm{M}^{\bullet+}+1\right]$. Anal. Calcd. for $\mathrm{C}_{16} \mathrm{H}_{10} \mathrm{~N}_{4}$ OS: C, 59.62; H, 3.13; N, 17.38; O, 9.93; S, 9.95. Found: C, 59.64; H, 3.16; N, 17.36; O, 9.95; S, 9.97.

3.3.4. 2-((1H-Benzimidazol-2-yl)thio)-3-(o-tolyl)acrylonitrile (2d). IR (KBr): $3400-2800 \mathrm{~cm}^{-1}$ (br, m, -NH-); ${ }^{1} \mathrm{H}-\mathrm{NMR}$ $\left(400 \mathrm{MHz}, \mathrm{DMSO}-\mathrm{d}_{6} / \mathrm{TMS}\right): \delta 8.15(\mathrm{~s}, 1 \mathrm{H}), 8.22(\mathrm{~d}, J=$ $4.2 \mathrm{~Hz}, 2 \mathrm{H}), 7.85-7.63(\mathrm{~m}, J=6.4 \mathrm{~Hz}, 2 \mathrm{H}), 7.62-7.45(\mathrm{~m}$, $J=6.3 \mathrm{~Hz}, 4 \mathrm{H}), 12.2(\mathrm{~s}, 1 \mathrm{H},-\mathrm{NH}-), 3.86$ (s, 3H), ${ }^{13} \mathrm{C} \mathrm{NMR:} \delta$ 140.2, 136.5, 133.2, 130.7, 127.4, 120.7, 117.4, 111.3 ppm; MS (CI): $m / z 292\left[\mathrm{M}^{\bullet+}+1\right]$. Anal. Calcd. for $\mathrm{C}_{17} \mathrm{H}_{13} \mathrm{~N}_{3} \mathrm{~S}$ : C, 70.08; $\mathrm{H}$, 4.50; N, 14.42; S, 11.00. Found: C, 70.11; H, 4.53; N, 14.45; S, 11.04 .

3.3.5. 2-((1H-Benzimidazol-2-yl)thio)-3-(p-tolyl)acrylonitrile (2e). IR (KBr): $3400-2800 \mathrm{~cm}^{-1}$ (br, m, -NH-); ${ }^{1} \mathrm{H}-\mathrm{NMR}$ $\left(400 \mathrm{MHz}, \mathrm{DMSO}-\mathrm{d}_{6} / \mathrm{TMS}\right): \delta 8.23(\mathrm{~s}, 1 \mathrm{H}), 8.25(\mathrm{~d}, J=$ $4.1 \mathrm{~Hz}, 2 \mathrm{H}), 7.75-7.53(\mathrm{~m}, J=6.3 \mathrm{~Hz}, 2 \mathrm{H}), 7.65-7.42(\mathrm{~m}$, $J=6.2 \mathrm{~Hz}, 4 \mathrm{H}), 12.2(\mathrm{~s}, 1 \mathrm{H},-\mathrm{NH}-), 3.72(\mathrm{~s}, 3 \mathrm{H}),{ }^{13} \mathrm{C} \mathrm{NMR:} \delta$ $140.4,136.7,133.5,131.7,126.4,120.7,116.4,110.3$ ppm; MS (CI): $m / z 292\left[\mathrm{M}^{\bullet+}+1\right]$. Anal. Calcd. for $\mathrm{C}_{17} \mathrm{H}_{13} \mathrm{~N}_{3} \mathrm{~S}$ : C, 70.08; $\mathrm{H}$, 4.50 ; N, 14.42; S, 11.00. Found: C, 70.11; H, 4.53; N, 14.45; S, 11.04 .

3.3.6. 2-((1H-Benzimidazol-2-yl)thio)-3-(4-hydroxyphenyl)acrylonitrile (2f). IR (KBr): $3400-2800 \mathrm{~cm}^{-1}$ (br, m, $\left.-\mathrm{NH}-\right) ;{ }^{1} \mathrm{H}-$ NMR (400 MHz, DMSO-d $\left.{ }_{6} / \mathrm{TMS}\right): \delta 8.20(\mathrm{~s}, 1 \mathrm{H}), 8.28$ (d, $J=4.0 \mathrm{~Hz}, 2 \mathrm{H}), 7.78-7.59(\mathrm{~m}, J=6.0 \mathrm{~Hz}, 2 \mathrm{H}), 7.60-7.48$ $(\mathrm{m}, J=6.0 \mathrm{~Hz}, 4 \mathrm{H}), 10.0\left(\mathrm{~s}, 1 \mathrm{H},-\mathrm{NH}-, \mathrm{D}_{2} \mathrm{O}\right.$ exchangeable), 12.5 (s, $1 \mathrm{H},-\mathrm{OH}, \mathrm{D}_{2} \mathrm{O}$ exchangeable) ${ }^{13} \mathrm{C}$ NMR: $\delta 140.6$, 136.9, 133.7, 132.7, 124.4, 121.7, 115.4, 111.3 ppm; MS (CI): $m / z$ $294\left[\mathrm{M}^{\circ+}+1\right]$. Anal. Calcd. for $\mathrm{C}_{16} \mathrm{H}_{11} \mathrm{~N}_{3} \mathrm{OS}$ : C, 65.51; $\mathrm{H}, 3.78$; N, 14.32; O, 5.45; S, 10.93. Found: C, 65.55; H, $3.76 ; \mathrm{N}, 14.34 ; \mathrm{O}, 5.46 ; \mathrm{S}, 10.95$.

3.3.7. 2-((1H-Benzimidazol-2-yl)thio)-3-(2-hydroxyphenyl)acrylonitrile (2g). IR (KBr): $3400-2800 \mathrm{~cm}^{-1}$ (br, m, -NH-); ${ }^{1} \mathrm{H}-$ NMR (400 MHz, DMSO-d d $_{6}$ TMS): $\delta 8.16(\mathrm{~s}, 1 \mathrm{H}), 8.22$ (d, $J=$ $4.3 \mathrm{~Hz}, 2 \mathrm{H}), 7.72-7.56(\mathrm{~m}, J=6.2 \mathrm{~Hz}, 3 \mathrm{H}), 7.58-7.28(\mathrm{~m}, J=$ $6.0 \mathrm{~Hz}, 4 \mathrm{H}), 10.2$ (s, $1 \mathrm{H},-\mathrm{NH}-, \mathrm{D}_{2} \mathrm{O}$ exchangeable); 12.3 (s, $1 \mathrm{H},-\mathrm{OH}, \mathrm{D}_{2} \mathrm{O}$ exchangeable); ${ }^{13} \mathrm{C}$ NMR: $\delta$ 141.6, 135.9, 132.7, 131.7, 123.4, 120.7, 114.4, 110.3 ppm; MS (CI): $m / z 294\left[\mathrm{M}^{\bullet+}+\right.$ 1]. Anal. Calcd. for $\mathrm{C}_{16} \mathrm{H}_{11} \mathrm{~N}_{3}$ OS: C, 65.51; H, 3.78; N, 14.32; O, 5.45; S, 10.93. Found: C, 65.55; H, 3.76; N, 14.34; O, 5.46; S, 10.95 .

3.3.8. 2-((1H-Benzimidazol-2-yl)thio)-3-(3,4-dimethoxyphenyl)acrylonitrile (2h). IR (KBr): $3400-2800 \mathrm{~cm}^{-1}$ (br, m, $\mathrm{NH}-$ ); ${ }^{1} \mathrm{H}-\mathrm{NMR}\left(400 \mathrm{MHz}, \mathrm{DMSO}-\mathrm{d}_{6} / \mathrm{TMS}\right): \delta 8.52(\mathrm{~s}, 1 \mathrm{H})$, $7.78(\mathrm{~d}, J=4.1 \mathrm{~Hz}, 2 \mathrm{H}), 7.77-6.92(\mathrm{~m}, J=6.2 \mathrm{~Hz}$, $2 \mathrm{H}), 7.56-6.94(\mathrm{~m}, J=6.0 \mathrm{~Hz}, 4 \mathrm{H}), 10.5(\mathrm{~s}, 1 \mathrm{H},-\mathrm{NH}-$ ), $3.26(\mathrm{~s}, 3 \mathrm{H}), 2.89(\mathrm{~s}, 3 \mathrm{H}),{ }^{13} \mathrm{C}$ NMR: $\delta$ 141.4, 135.5, 130.7, 130.2, 123.2, 119.7, 114.4, 109.3 ppm; MS (CI): $m / z 338$ $\left[\mathrm{M}^{\bullet+}+1\right]$. Anal. Calcd. for $\mathrm{C}_{16} \mathrm{H}_{11} \mathrm{~N}_{3} \mathrm{OS}$ : C, 64.08; H, 4.48;
N, 12.45; O, 9.48; S, 9.50. Found: C, 64.10; H, 4.46; N, 12.47; O, $9.45 ; \mathrm{S}, 9.52$.

\section{Conclusion}

In summary, we have developed a simple, atom economical, highly efficient, environmentally benign procedure for the synthesis of benzimidazolylacrylonitriles using simple ultrasonochemical method using water as a green solvent. Further, the reaction was successful without decrease in product yield. The advantages of the present procedure are the generally good yields, mild and environmentally benign inexpensive bench top chemicals, or solvents and operational simplicity.

\section{Conflict of Interests}

The authors declare that there is no conflict of interests regarding the publication of this paper.

\section{References}

[1] T. Eicher, The Chemistry of Heterocycles: Structure, Reactions, Syntheses, and Applications, vol. 2, Wiley-VCH, Weinheim, Germany, 2003.

[2] J. P. Lorimer, Applied Sonochemistry: Uses of Power Ultrasound in Chemistry and Processing, vol. 1, Wiley-VCH, Weinheim, Germany, 2002.

[3] L. Song, S. Zhang, X. Wu, and Q. Wei, "Synthesis of porous and trigonal $\mathrm{TiO}_{2}$ nanoflake, its high activity for sonocatalytic degradation of rhodamine B and kinetic analysis," Ultrasonics Sonochemistry, vol. 19, no. 6, pp. 1169-1173, 2012.

[4] R. Ghahremanzadeh, F. Fereshtehnejad, P. Mirzaei, and A. Bazgir, "Ultrasound-assisted synthesis of 2,2' -(2-oxoindoline3,3-diyl)bis( $1 H$-indene-1,3(2H)-dione) derivatives," Ultrasonics Sonochemistry, vol. 18, no. 1, pp. 415-418, 2011.

[5] E. Kowsari and M. Mallakmohammadi, "Ultrasound promoted synthesis of quinolines using basic ionic liquids in aqueous media as a green procedure," Ultrasonics Sonochemistry, vol. 18, no. 1, pp. 447-454, 2011.

[6] J.-Y. He, H.-X. Xin, H. Yan, X.-Q. Song, and R.-G. Zhong, "Convenient ultrasound-mediated synthesis of 1,4-diazabutadienes under solvent-free conditions," Ultrasonics Sonochemistry, vol. 18, no. 1, pp. 466-469, 2011.

[7] J.-T. Li, Y. Yin, and M.-X. Sun, "An efficient one-pot synthesis of 2,3-epoxyl-1,3-diaryl-1-propanone directly from acetophenones and aromatic aldehydes under ultrasound irradiation," Ultrasonics Sonochemistry, vol. 17, no. 2, pp. 363-366, 2010.

[8] A. Bazgir, S. Ahadi, R. Ghahremanzadeh, H. R. Khavasi, and P. Mirzaei, "Ultrasound-assisted one-pot, three-component synthesis of spiro[indoline-3,4'-pyrazolo[3,4-b]pyridine]$2,6^{\prime}\left(1^{\prime} \mathrm{H}\right)$-diones in water," Ultrasonics Sonochemistry, vol. 17, no. 2, pp. 447-452, 2010.

[9] K. S. Suslick, "Sonochemistry," Science, vol. 247, no. 4949, pp. 1439-1445, 1990.

[10] H. Fillion and J. L. Luche, Synthetic Organic Sonochemistry, Plenum Press, New York, NY, USA, 1998.

[11] A. Gedanken, "Using sonochemistry for the fabrication of nanomaterials," Ultrasonics Sonochemistry, vol. 11, no. 2, pp. 47$55,2004$. 
[12] C. H. Heathcock, R. C. D. Brown, and T. C. Norman, "Synthesis of Petrosins C and D," The Journal of Organic Chemistry, vol. 63, no. 15, pp. 5013-5030, 1998.

[13] X. Wang, A. Guram, E. Bunel, G.-Q. Cao, J. R. Allen, and M. M. Faul, "Palladium-catalyzed one-pot synthesis of 2-alkyl-2arylcyanoacetates," Journal of Organic Chemistry, vol. 73, no. 4, pp. 1643-1645, 2008.

[14] S. D. Walker, C. J. Borths, E. Divirgilio et al., "Development of a scalable synthesis of a GPR40 receptor agonist," Organic Process Research and Development, vol. 15, no. 3, pp. 570-580, 2011.

[15] S. J. Mukarram, R. Khan, and R. Yadav, "Efficient method for the manufacture of (E) - Entacapone polymorphic form A," United States Patent No.: 0004935, 2007.

[16] U. R. Pratap, D. V. Jawale, R. A. Waghmare, D. L. Lingampalle, and R. A. Mane, "Synthesis of 5-arylidene-2,4thiazolidinediones by Knoevenagel condensation catalyzed by baker's yeast," New Journal of Chemistry, vol. 35, no. 1, pp. 49-51, 2011.

[17] A. M. A. Al-Kadasi and G. M. Nazeruddin, "Ultrasound assisted catalyst-free one-pot synthesis of bis-coumarins in neat water," International Journal of Chemical Sciences, vol. 10, no. 1, pp. 324330, 2012.

[18] J. Safari, S. H. Banitaba, and S. D. Khalili, "Ultrasoundpromoted an efficient method for one-pot synthesis of 2amino-4,6-diphenylnicotinonitriles in water: a rapid procedure without catalyst," Ultrasonics Sonochemistry, vol. 19, no. 5, pp. 1061-1069, 2012.

[19] V. R. Reddy, P. K. Dubey, and S. S. Rao, "Synthesis of $\alpha-$ benzylthiobenzimidazole acetonitriles and their chemoselective reduction of the double bond with $\mathrm{NaBH}_{4}$, Journal of Heterocyclic Chemistry. In press. 

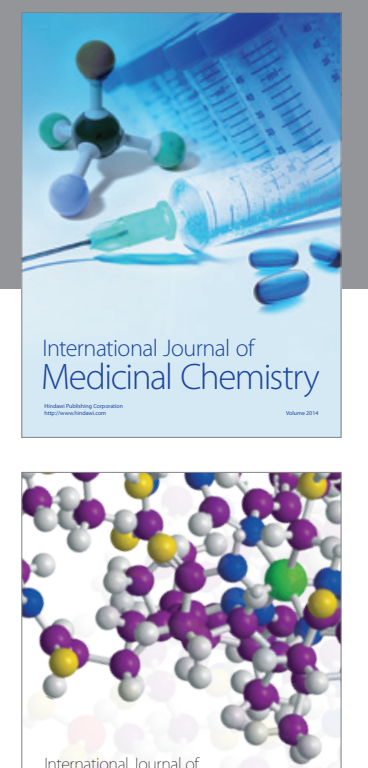

\section{Carbohydrate} Chemistry

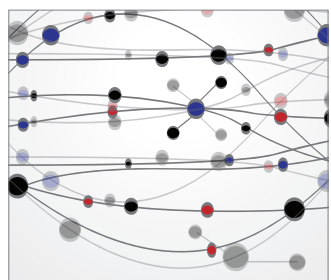

The Scientific World Journal
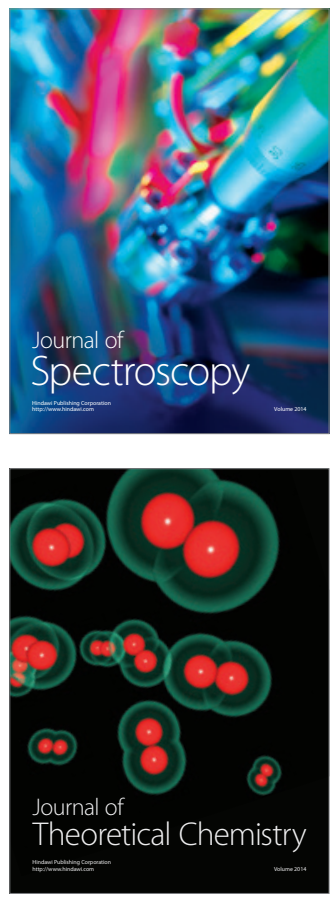
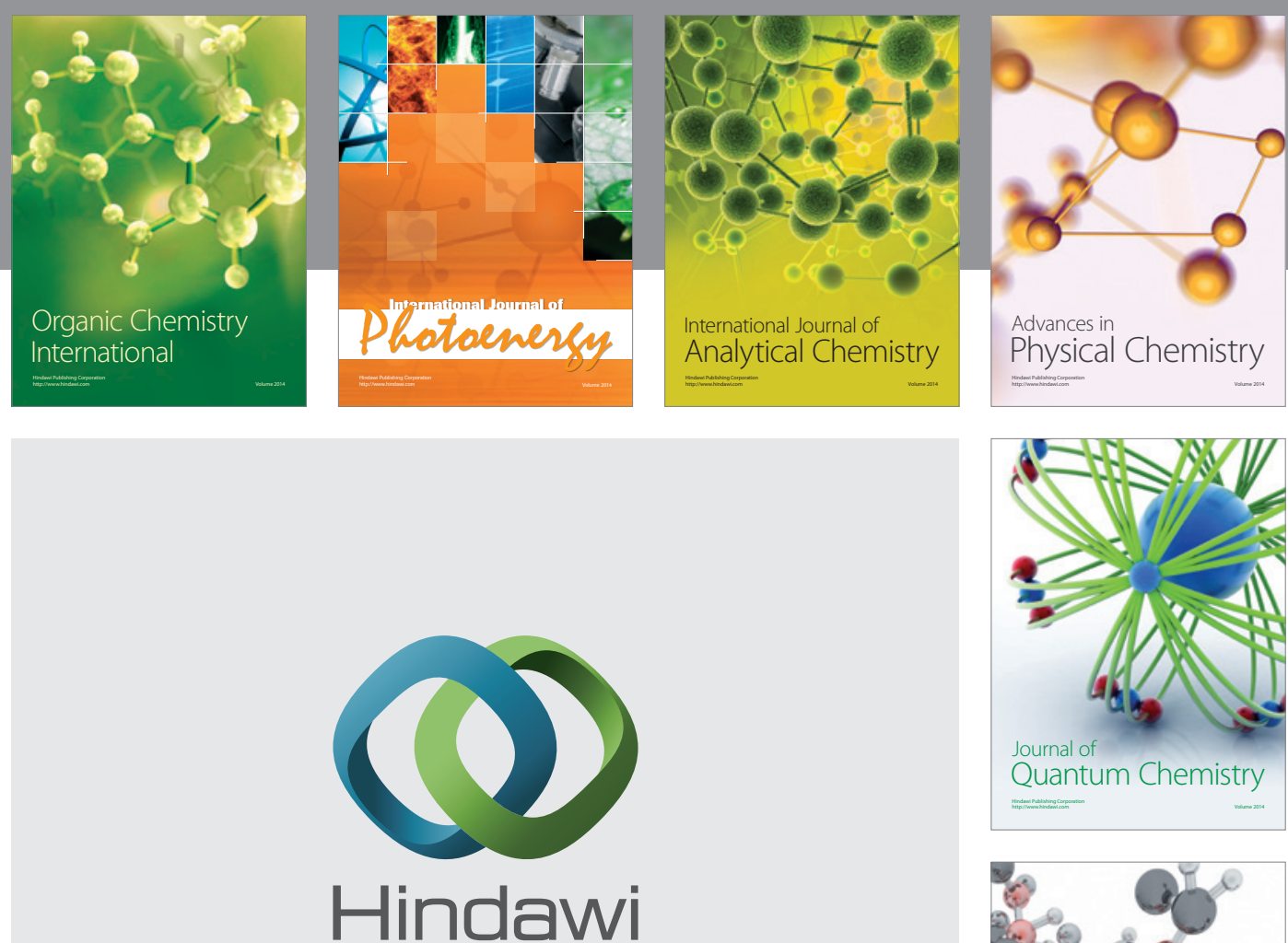

Submit your manuscripts at

http://www.hindawi.com

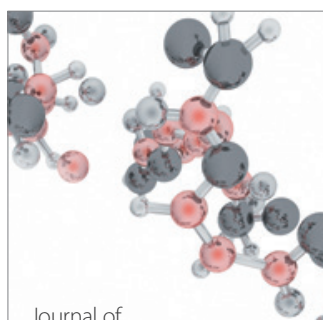

Analytical Methods

in Chemistry

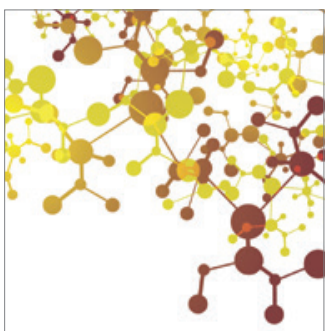

Journal of

Applied Chemistry

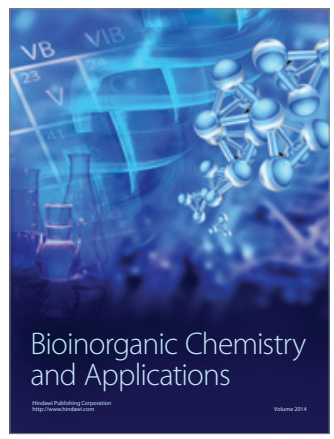

Inorganic Chemistry
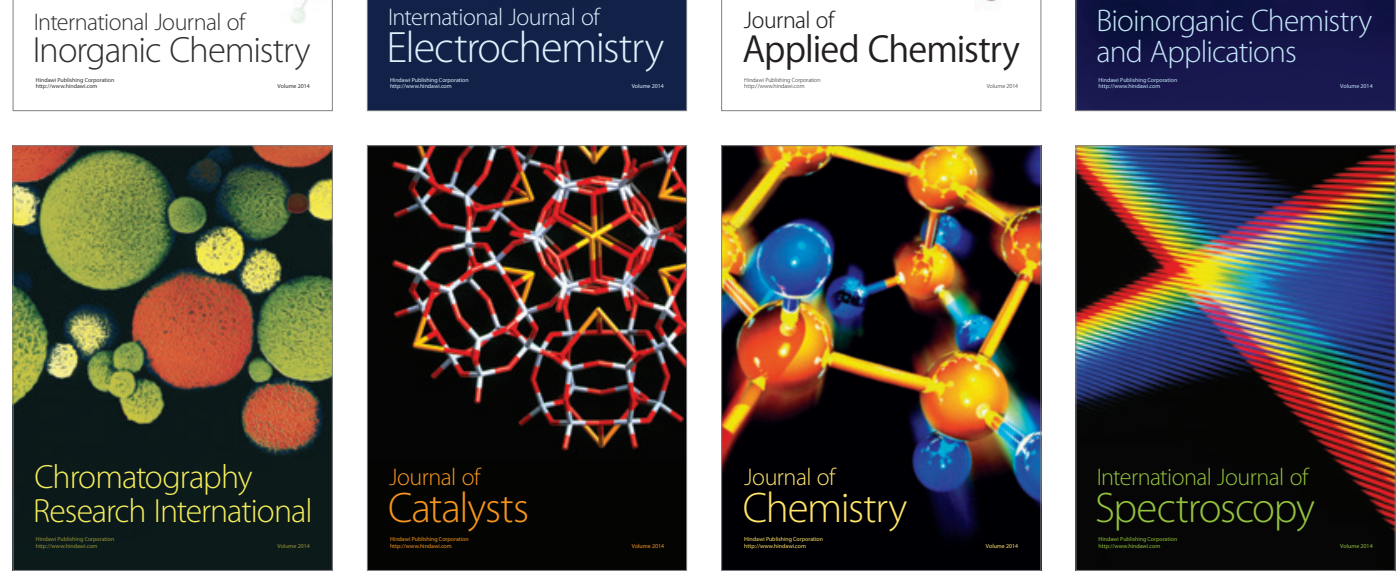